\title{
Pioneers of the New Physics
}

Stuart Sharrock puts the achievement

\section{of Richter and Ting into perspective}

THE discovery of stable elementary particles of high mass has opened up a new era in experimental and theoretical particle physics. For their ploneering work in this discovery Professor Burton Richter of the Stanford Linear Accelerator Center (SLAC) and Professor Samuel Ting of the Massachusetts Institute of Technology have been jointly awarded the 1976 Nobel Prize for physics.

The existence of a new particle was announced simultaneously by the two experimental teams in November 1974. It was observed independently by Ting's group working on the proton accelerator at the Brookhaven National Laboratory (BNL), and by Richter's team at the electron-positron storage ring facility at SLAC. The new particle, called $J$ at BNL and $\psi$ at SLAC, is a neutral meson of high mass, $3.1 \mathrm{GeV}$, and with the amazingly narrow width of $100 \mathrm{keV}$. It is difficult to detect with proton machines, and its discovery at BNL was a great feat of experimentation. In contrast it is copiously produced in electron-positron annihilation, although its narrow width makes it elusive. Within a few days of the announcement of the $J / \psi$ the SLAC team had discovered a further state, the $\psi^{\prime}$ at $3.7 \mathrm{GeV}$.

The existence of stable particles of high mass was unexpected. The $\mathbf{J} / \psi$ is a spin 1 particle and is more than twenty times heavier than the lightest such particle, the pion. The $J / \psi$ should rapidly decay into lighter known particles, yet its lifetime (related to its width through the uncertainty principle) is about 1,000 times longer than expected. Furthermore the $\mathrm{J} / \psi$ does not fit into the well-established three-quark classification scheme. A new law of nature is therefore necessary to explain its decay properties and extremely long lifetime.

of the four known forces between particles only the electromagnetic interaction is understood. Both Richter and Ting have in the past contributed to this understanding by demonstrating the validity of the theory down to distances of the order of $10^{-14} \mathrm{~cm}$. Attempts at a unified field theory for the weak and electromagnetic interactions based on the principle of 'gauge invariance', in which an additional symmetry is assumed to exist between the two interactions, have had some success in recent years, particularly with the prediction and subsequent observation of the neutral current weak interactions. The theories require the existence of a fourth quark, distinguished by the name 'charm'. This new quantum number has successfully explained the $J / \psi$ particle as a bound state of a charmed quark and a charmed anti-quark.

If the charmed quark is heavy then the long lifetime of the $J / \psi$ is explained. The bound state of charmanticharm is similar to that of the $\mathrm{e}^{+} \mathrm{e}$ system in positronium, and is called charmonium. A number of energy levels are expected in this model and many states have now been found fitting well into the predicted level scheme. The investigation of charmonium promises to play a key role in the understanding of strong interactions by providing information on the force acting between quarks.

Particles are classified as hadrons if they have strong interactions and leptons if they do not. The addition of a charmed quark to the three conventional quarks provides a set of building blocks from which all known hadrons can be constructed. It also provides a symmetry between leptons and quarks, and there are now four of each with intcractions that can be expressed in terms of the same set of mathematical rules. The detection of the new particles has given rise to a new branch or hadron physics which could lead to a better understanding of the symmetries governing the structure of elementary particles. Just as the discovery of neutral currents may help to unify the weak and electromagnetic interactions, the concept of a charmed quark could well indicate links between the weak and strong interactions.

Professor Ting's experiment at BNL was part of a systematic and precise study of lepton pairs produced in hadron-hadron collisions. This study was started in 1972 and, to cover the mass region 1-50 GeV, involved experiments at BNL, the ISR in Geneva and the electron synchrotron DESY in Hamburg. One of the aims was to search for long-lived particles decaying into lepton pairs. These experiments are very difficult as the rate of electromagnetic pair production in hadronhadron collisions is extremely low compared to the rate of production of hadron pairs. The experiment had to reject hadron pairs by a factor of at least 100 million. During the period August-October 1974 the Ting group

\author{
Sorry, for copyright \\ reasons some images \\ on this page may not \\ be available online
}

\section{Samuel Ting}

had seen a narrow peak in the effective mass distribution of electron pairs, and had performed many detailed checks to prove that the peak was a real particle. The announcement of the discovery was withheld at first while the experiment was modified to search for possible further new phenomena. The inevitable rumours began to circulate and the group decided to publish at the beginning of November. Around this time the new particle was also seen at SLAC and the results of the two experiments were published simultaneously.

At SLAC, Richter's team had been working with the immense magnetic detector at the electron-positron storage ring complex (SPEAR). They had compiled a large volume of data on the total cross-section for $\mathrm{e}^{+} \mathrm{e}^{-}$annihilation as a function of energy. On checking through these data an anomaly was observed at one particular energy setting: inconsistent values of the total cross-section were obtained in measurements taken at the same nominal energy. The group decided this anomaly had to be cleared up, and at the beginning of November they decided to investigate the energy region in detail. Within hours of starting an extremely narrow peak in the 
cross-section had been seen. This effect was so strong and clear that the group immediately sat down and wrote the draft of their paper announcing the discovery of the new particle. The following day Richter and Ting met at a committee meeting and to their mutual astonishment started to tell each other about the interesting physics results they had found.

Burton Richter was born in 1931 in New York. He decided to become a scientist as a child in high school, a physicist as an undergraduate, and a particle physicist after obtaining his $\mathrm{PhD}$ from MIT in 1956. Richter, always fascinated by electrons, sought a job at SI.AC where he performed experiments demonstrating the range of validity of quantum electrodynamics

The Nobel prizes (2): Chemistry

\section{Boranes surgery}

\section{A Special Correspondent looks at the work of William Lipscomb}

$T^{T}$ is no surprise to chemists that Bill I Lipscomb should have been awarded the Nobel Prize for his beautiful work on the boron hydrides. The layman may, however, be baffled, wondering what these oddly-named entities might be, and why they should have merited years of study by a leading crystallographer who is no less well known for his more recent work on the structure of enzymes.

The story gocs back more than half a century, to the time when the great inorganic chemist Alfred Stock reported that the simplest compound of boron and hydrogen is not $\mathrm{BH}_{3}$, as one would expect by analogy with $\mathrm{BF}_{3}$, but its dimer $\mathrm{B}_{2} \mathrm{H}_{6}$. The trouble with $\mathrm{B}_{2} \mathrm{H}_{6}$, and with all the other boron hydrides which he described, was its "electron deficiency". If the molecule had an cthane-like geometry (as the evidence of electron diffraction was held to indicate) it would need 7 electron-pair bonds to hold its atoms together, but only 12 valency electrons were available for the purpose. This ominous crack in orthodox valency theory was papered over by Linus Pauling, who advanced special reasons why the B-H bond should be able to make do with less than 2 electrons, and proposed structures for the higher boron hydrides in line with this idea.

In 1943 Longuet-Higgins and Bell showed that the chemical and spectroscopic evidence on B. $_{2} \mathrm{H}_{6}$ harmonised less well with an ethane-like structure than with a bridge structure, in which down to very small distances. He has long been a proponent of the virtues of electron-electron scattering and was instrumental in the commissioning of the $\mathrm{e}^{-} \mathrm{e}^{-}$colliding beam machine at SLAC. During this time he designed an $\mathrm{e}^{+} \mathrm{e}^{-}$storage ring and worked for ten years to create the complex known as SPEAR. His team working on SPEAR began experiments in 1973 culminating in the discovery of the $\psi$ family of particles and the subsequent measurement of their properties.

Samuel Ting was born in 1936 in Michigan but returned to China with his parents when he was two months old. At the age of 20 he returned to America with $\$ 100$, very little knowledge of English and a determination to go to university. Within a short time he had obtained a degree and a PhD from the University of Michigan and decided to become an experimental physicist. After a year at CERN he returned to teach at Columbia University in 1965. At Columbia he proposed an experiment at DESY to check a recent experimental result which appeared to show a violation of quantum electrodynamics. This was accepted, and in 1966 he began a long association with DESY in which he performed experiments extending the range of validity of quantum electrodynamics and determining the properties of vector mesons. His work over the last decade on lepton production culminated in the discovery of the $J / \psi$ by its decay into electron pairs. two of the $\mathrm{H}$ atoms are situated in the middle of the molecule. It appeared that $\mathrm{B}_{2} \mathrm{H}_{6}$ manages to hold together, not by starving all its bonds of electrons but by sharing two electron pairs between the two sides of the central bridge. The bridge structure was confirmed soon afterwards by W. C. Price, and in 1949 it was proposed that one should regard $B_{i} H_{i i}$ as containing two "liaisons triatomiques", or "3-centre bonds", neatly describable in terms of localised molecular orbitals. But the higher boron hydrides remained a puzzle.

At this point the crystallographers grasped the initiative, and Kasper, Lucht and Harker led off with a definitive determination of the molecular structure of crystalline $\mathbf{B}_{10} \mathrm{H}_{14}$. Their results if anything deepened the mystery: the bonding in this molecule clearly involved principles more general than those which had bcen advanced for $\mathrm{B}_{2} \mathrm{H}_{6}$, and it became clear that a full understanding of these principles must await the collection of structural evidence on the other boron hydrides.

It was Lipscomb who took up this challenge. Undaunted by the pathological instability of most boron hydrides and the acute difficulty of "seeing" hydrogen atoms by X-ray diffraction, he and his colleagues completed a sequence of brilliant experimental studies of the hydrides described by Stock, and of others isolated in his own laboratory. A pattern soon

\section{Sorry, for copyright \\ reasons some images on this page may not be available online}

\section{William Lipscomb}

began to emerge which could be interpreted in terms of "3-centre bonds" of various novel kinds, together with "many-centre bonds", for whose possible existence there were solid grounds in molecular orbital theory. Simultaneous theoretical studies in England and by Lipscomb's group predicted the existence of a stable ion $\mathrm{B}_{12} \mathrm{H}_{12}{ }^{--}$ with icosahedral symmetry.

The preparation of the potassium salt, and the subsequent determination of its structure by Wunderlich and Lipscomb in 1960 , set the final seal on the molecular orbital theory of the bonding in these remarkable substances. The fascinating work of Lipscomb and his colleagues is a story of the fruitful interplay between imaginative theory and skilled experiment, leading to a genuine "breakthrough" in our understanding of the forces which bind atoms together. No longer is there a dichotomy between the chemist's "covalent" bond and the "metallic" bond of the physicist: Lipscomb's work showed us that at least one element succeeds, in its hydrites, in forming both kinds of bond within the same molecular species. 